\title{
Severe odontogenic infection: An emergency. Case report
}

\author{
Marcelo Guzmán-Letelier ${ }^{1}$, Claudia Crisosto-Jara ${ }^{2}$, Camilo Diaz-Ricouz ${ }^{3}$, Miguel Peñarrocha-Diago ${ }^{4}$, \\ David Peñarrocha-Oltra 5
}

\author{
${ }^{1}$ DDS, Maxillofacial Surgeon, Associate Professor, San Sebastián University Dental School. Valdivia, Chile Maxillofacial Sur- \\ geon, Hospital Base Valdivia, Chile \\ ${ }^{2}$ DDS, Dental surgeon. Associate Professor, San Sebastián University Dental School. Valdivia, Chile \\ ${ }^{3}$ DDS, Oral Surgery Collaborator, San Sebastián University Dental School. Valdivia, Chile \\ ${ }^{4} \mathrm{MD}, \mathrm{PhD}$, DDS, Chairman of Oral Surgery, Valencia University Medical and Dental School, Valencia, Spain \\ ${ }^{5} \mathrm{PhD}$, DDS, Associate Professor Department of Stomatology, Valencia University Medical and Dental School. Valencia, Spain
}

Correspondence:

General Lagos 1163

Edificio B ler piso

Facultad de Odontología Universidad San Sebastian

Valdivia, Chile

drmguzmanl@gmail.com

Received: 10/06/2016

Accepted: 02/08/2016

\begin{abstract}
Guzmán-Letelier M, Crisosto-Jara C, Diaz-Ricouz C, Peñarrocha-Diago M, Peñarrocha-Oltra D. Severe odontogenic infection: An emergency. Case report. J Clin Exp Dent. 2017;9(2):e319-24.

http://www.medicinaoral.com/odo/volumenes/v9i2/jcedv9i2p319.pdf
\end{abstract}

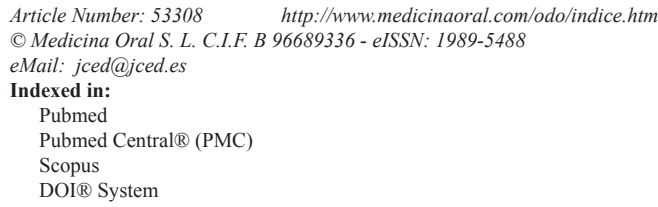

\begin{abstract}
Odontogenic infections (OI) are a major reason for consultation in dental practice. They affect people of all ages, and most of them respond well to current medical and surgical treatments. However, some OI can spread to vital and deep structures, overcome the host immune system - especially in diabetic, immunocompromised or weakened patients - and even prove fatal. Ludwig's angina is a severe form of diffuse cellulitis that can have an acute onset and spread very rapidly, bilaterally affecting areas of the head and neck, and may prove life threatening. A case of severe dental infection is presented in which emphasis is placed on the importance of airway maintenance, followed by surgical decompression under adequate antibiotic coverage.
\end{abstract}

Key words: Ludwig's angina, severe odontogenic infection, surgical decompression, dental infection.

\section{Introduction}

Odontogenic infections (OI) are quite frequent, and usually can be resolved by local medical-surgical means - though in some cases they may become complicated and result in important morbidity-mortality (1). Odontogenic infections are generally secondary to pulp necrosis, periodontal disease, pericoronitis, apical lesions or complications of certain dental procedures (2).

The spread of an infection depends on the balance between the patient condition and microbial factors. The virulence of germs, along with the local and systemic conditions of the patient, determine host resistance $(3,4)$. Systemic alterations favoring the spread of infection can be observed in situations such as HIV/AIDS disease, decompensated diabetes mellitus, immune depression, alcoholism or weakened states $(1,3,4)$.

Ludwig's angina is a head and neck infection characterized by rapid progression, with edema and necrosis of the soft tissues of the neck and floor of the mouth, and is associated to a high mortality rate (5). The disea- 
se involves progressive tumefaction of the soft tissues and simultaneous alteration of the sublingual, submandibular and submental spaces, with elevation and subsequent displacement of the tongue, which can eventually obstruct and collapse the respiratory tract $(5,6)$. Before the age of antibiotics, the mortality rate in patients with Ludwig's angina was over 50\% (6). With the introduction of antibiotics and improvements in imaging and surgical techniques, the mortality rate has decreased to around $8 \%(6,7)$. However, in the past $10-15$ years there has been a re-emergence of difficulties in managing and treating such cases, probably as a consequence of resistance to antibiotics caused by indiscriminate use, and progressive aging of the population associated to nontransmissible chronic disorders such as diabetes mellitus $(3,4)$.

The location of the infectious process in the anatomical spaces of the buccofacial area determines the risk of compromising the respiratory tract and of affecting vital structures and organs (5). Flyn et al. recently simplified classification of the severity of OI, assigning a numerical score of 1 to 4 (mild, moderate, severe, extremely severe) to the anatomical spaces, according to the degree of impairment of the respiratory tract and/or vital structures such as the mediastinum, heart or contents of the cranial cavity (2). Increased severity of the infection and the appearance of complications prolong hospital stay, complicate surgical management, and place an increased demand upon Special Care Units (SCU). In this regard, the identification of risk factors associated to increased severity may be essential in order to establish early diagnosis and treatment (6-9).

We describe a case of severe odontogenic infection, and establish correlations between the disease and systemic risk factors such as diabetes mellitus and possible resistance to empirical antibiotic treatment.

\section{Case Report}

A 42-year-old male consulted due to sudden, progressive and painful tumefaction in the left submandibular region during the last 48 hours. The disease history revealed type 2 diabetes treated with glibenclamide ( $50 \mathrm{mg}$ /day), and arterial hypertension. Both conditions had not been followed-up on over the last 12 months. The patient suffered diabetic retinopathy and had been treated for lung tuberculosis. He had been initially diagnosed and treated by his dentist for symptoms of pericoronitis affecting tooth 3.8 , with the prescription of oral antibiotics (amoxicillin $500 \mathrm{mg}+$ clavulanic acid $125 \mathrm{mg} 3$ times a day) and oral nonsteroidal antiinflammatory drugs (ibuprofen $400 \mathrm{mg} 3$ times a day). Following limited response to the initial medical treatment, the patient decided to consult the maxillofacial surgery unit.

At consultation, the patient was found to be conscious, with asthenia, dehydration, fever $\left(38.5^{\circ} \mathrm{C}\right)$, dysphagia, severe trismus and submaxillary adenopathies. He also presented tachycardia and tachypnea $(23 \mathrm{rpm})$ associated to inspiratory stridor, and with SatO2 93\%. The patient showed marked facial asymmetry, with a painful indurated tumefaction in the left submandibular region, without clear boundaries.

Despite the difficulty in carrying out the intraoral examination because of the trismus, a painful retromolar tumefaction was identified in relation to third molar 3.8 , extending to the ipsilateral floor of the mouth. The panoramic X-ray study (Fig. 1) revealed the mentioned third molar semi-impacted in a distoangular position. A phlegmon on the floor of the mouth (Ludwig's angina) was diagnosed, secondary to acute suppurative pericoronitis of tooth 3.8 .

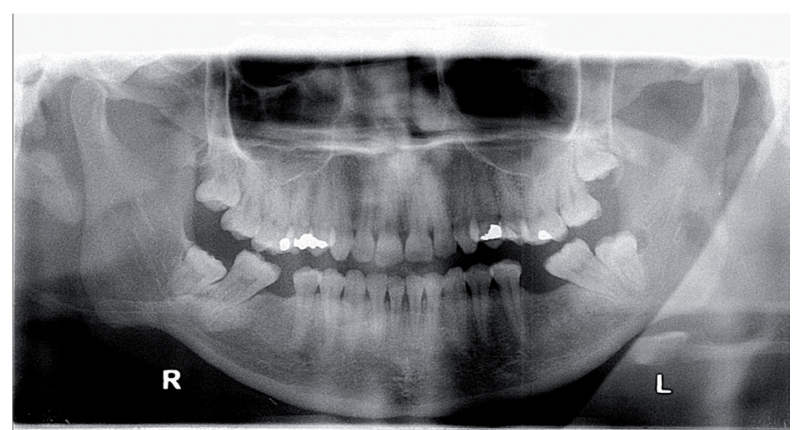

Fig. 1. Panoramic X-ray view at initial presentation. Note the irregular pericoronal radiolucency associated to partial bony impacted tooth 3.8 in vertical position (2B Pell and Gregory). Pericoronal distal widened space compatible with paradental inflammatory cyst.

Due to the severity of the symptoms, the patient was hospitalized and obtained informed consent for registry and treatment physician-surgical. Empirical intravenous antibiotic therapy (clindamycin $600 \mathrm{mg}$ every 8 hours and ceftriaxone $2 \mathrm{~g}$ every 24 hours). Upon admission the patient presented leukocytosis $\left(20,000\right.$ cells $\left./ \mathrm{mm}^{3}\right)$, a C-reactive protein concentration of $300 \mathrm{mg} / \mathrm{l}$, blood glucose $325 \mathrm{mg} / \mathrm{dl}$ and glycosylated hemoglobin (HbA1c) $17.6 \%$. Treatment with insulin was prescribed.

Within a few hours the clinical condition worsened, with a large edema developing in the floor of the mouth and breathing difficulties. Exploration was carried out via direct laryngoscopy, and an emergency tracheotomy was performed due to the impossibility of intubation and ventilation (Fig. 2). The patient was subsequently placed under protective mechanical ventilation, and was moved to the Intensive Care Unit (ICU) for the continuation of medical management and stabilization. Following a computed tomography scan of the head and neck (Fig. 3 ), he developed acute renal failure with a plasma creatinine concentration of $5.7 \mathrm{mg} / \mathrm{dl}$. On day four of admission, the causal tooth 3.8 was extracted and drained, and an extended cervicotomy was performed (Figs. 4,5). Cultures proved positive for Acinetobacter baumannii 


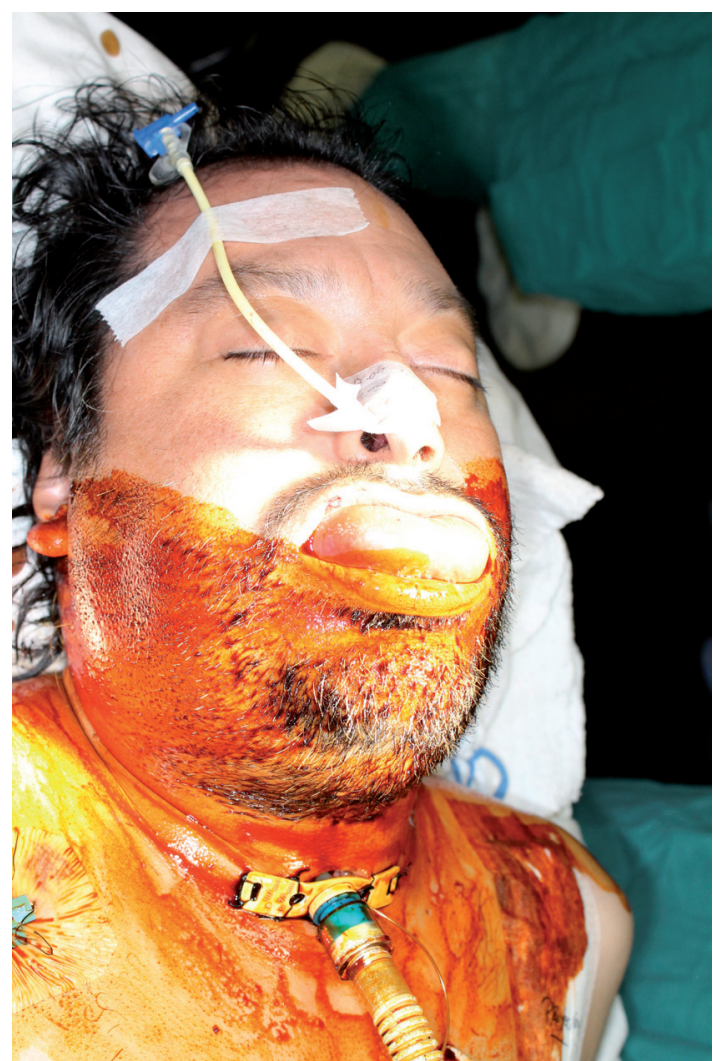

Fig. 2. Patient with diffuse severe cellulitis (Ludwig's angina); tracheostomy with intense swelling, simultaneous and bilateral submandibular, sublingual and submental space involvement, tongue elevation and protrusion, with total blockage of the upper airway, and protective mechanical ventilation.

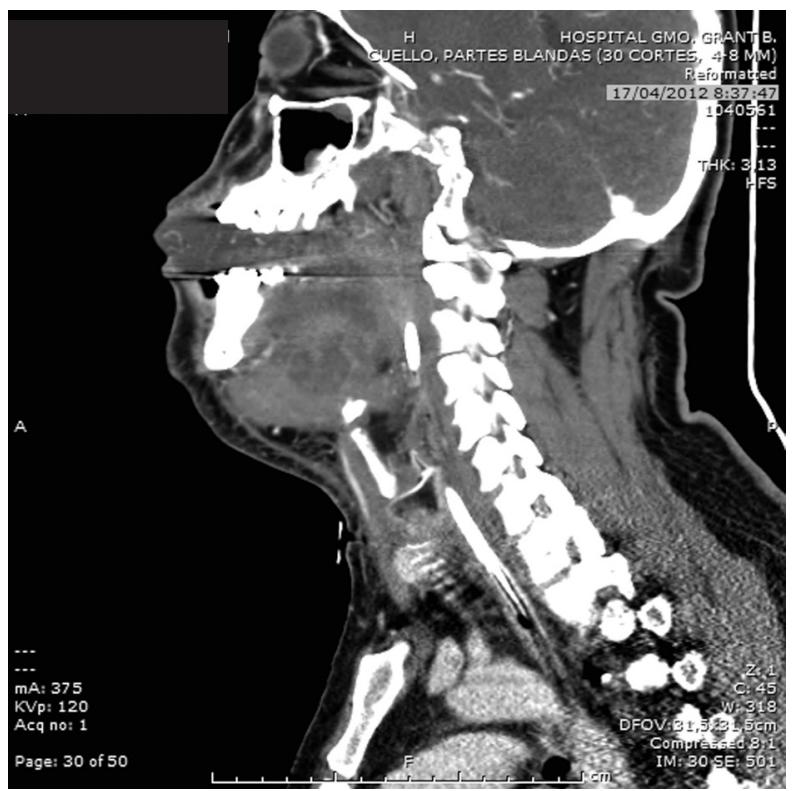

Fig. 3. Computed tomography. Sagittal section showing upper airway elevation and protrusion of the tongue showing airway impairment and a large hypodense collection suggestive of a diffuse Ludwig's angina infectious process.

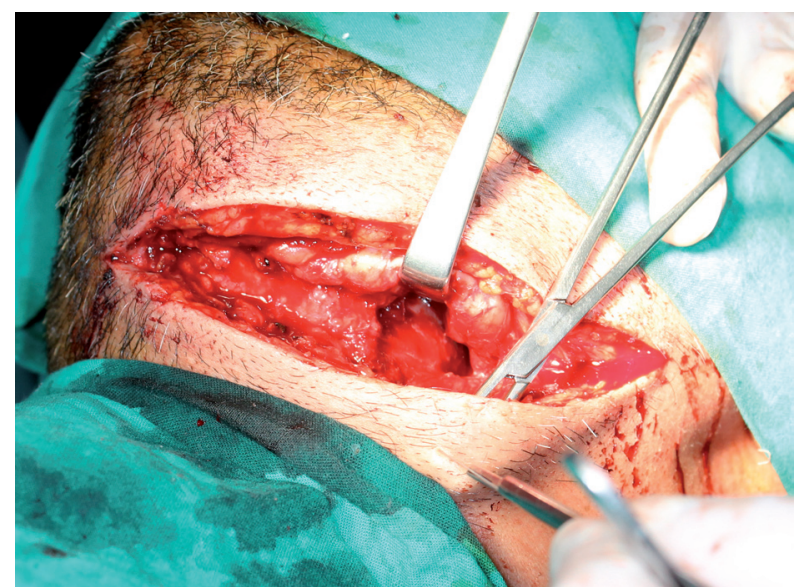

Fig. 4. Surgical exploration cervicotomy revealing neck tissue necrosis. Debridement and necrotic debris removal was carried out, with profuse surgical irrigation.

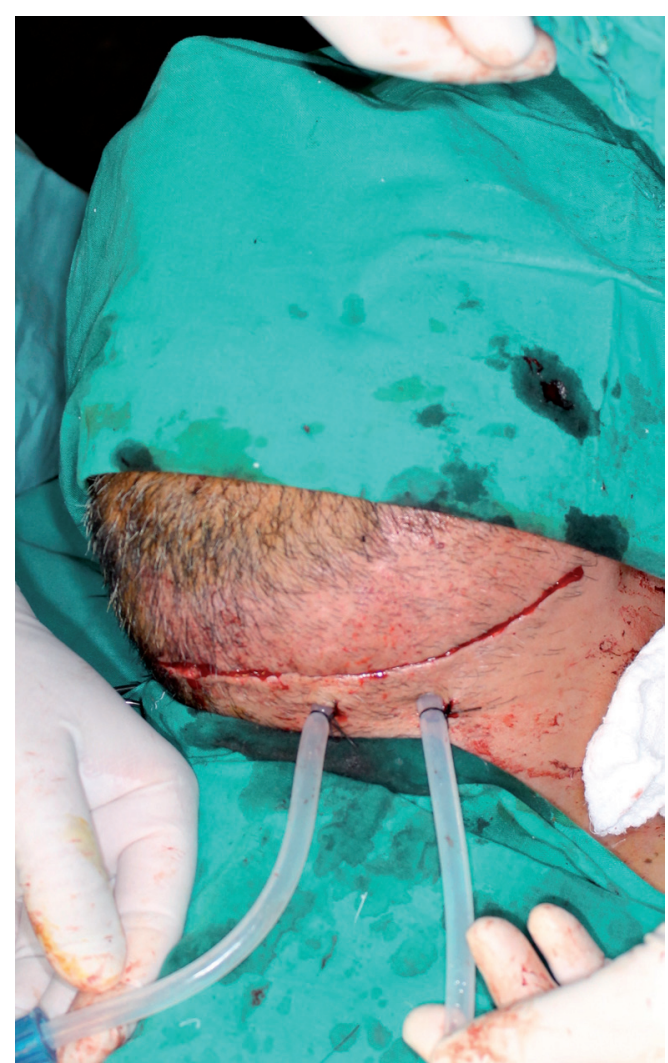

Fig. 5. Continuous drain: two tires are placed to facilitate irrigation and aspiration of the affected tissues with purulent content.

(AB) and methicillin-resistant Staphylococcus aureus (MRSA), and treatment with tigecycline was prescribed (50 mg every 12 hours i.v. during 14 days). The patient evolved favorably, with a decrease in inflammatory parameters and recovery of renal function. Extubation was carried out after two weeks, maintaining good respiratory and hemodynamic function, with a Glasgow coma score of 15 (Fig. 6). The inflammatory parameters im- 




Fig. 6. Extubated patient with removal of the tracheostomy tube Twenty days after surgery no obvious signs of infection are noted. Marked improvement of the patient condition, with stabilization and compensation of diabetes.

proved, with resolution of the fever. Spontaneous ventilation was restored, without the need for additional oxygen. On day 22 of hospital admission, the patient was in good general condition, hemodynamically stable, the surgical wound showed no signs of infection, and the inflammatory parameters were found to have normalized. Discharge was therefore decided, with ambulatory checkups after 7, 14 and 30 days.

\section{Discussion}

Ludwig's angina was first described in 1836 by the German physician Wilhelm Friedrich Von Ludwig as a severe infectious disease giving rise to rapidly evolving cellulitis in the floor of the mouth. The condition is potentially serious, as it may lead to sepsis, cause obstruction of the upper airway and produce edema of the epiglottis $(10,11)$.

The underlying infectious process may be of odontogenic or non-odontogenic origin. Odontogenic infection (OI) originates in teeth or in surrounding tissues, affecting the periapical bone from where it spreads towards either neighboring structures (continuous propagation) or structures located further away (distant propagation) (12-14). Odontogenic infections are the most frequent presentations, $70-90 \%$ originating from pulp necrosis, periodontal disease, pericoronitis, granulomas, apical cysts or complications of dental procedures. Non-odontogenic infections in turn are associated to maxillofacial fractures, submandibular sialoadenitis, infections of the salivary glands, tumor or cystic lesions, and infections of pharyngeal or tonsillar origin, among others $(2,11)$.

The literature describes odontogenic infections as the most common cause of head and neck ailments. Umeda et al. (6) presented 9 cases and reviewed the English language literature, documenting 125 infections of odontogenic origin. They reported periapical infections of the second and third mandibular molars as being the most frequent origin $(70-80 \%)$, due to the fact that the roots of these teeth typically extend beneath the mylohyoid muscle, producing infection that spreads into the submaxillary space, and from there to the sublingual and submental spaces, consecutively. Flynn et al. (2) published a study of 49 cases of severe odontogenic infections with involvement of the deep lying spaces. Of these cases, $68 \%$ were associated to inferior third molars, $22 \%$ to pericoronitis, and the rest to other mandibular posterior teeth. Our case is consistent with the origin described in the literature, involving a semi-impacted third mandibular molar with pericoronitis that evolved into a phlegmon in the floor of the mouth. Kurien et al. (15) carried out a comparative study of the causes of Ludwig's angina in children and adults. They identified a dental origin in $52 \%$ of the adults, and $39 \%$ suffered predisposing systemic diseases such as poorly managed diabetes, alcohol abuse or immunosuppression.

The affected anatomical spaces of the head and neck must be identified and classified according to their potential impact upon the respiratory tract and/or vital structures such as the mediastinum, heart or cranial content. Flynn et al. developed a severity scale (SS) for OI in which a numerical score of 1 to 4 is respectively assigned to mild, moderate, severe and extremely severe involvement of the anatomical spaces (Table 1). This numerical score closely relates anatomical space involvement to the risk of affecting the respiratory tract and vital structures. According to the mentioned classification, if a patient has more than one compromised space, a sum of all values is made. In our case, the patient showed involvement of the submaxillary space ( $\mathrm{SS}=2$ ), sublingual space $(\mathrm{SS}=2)$, submental space $(\mathrm{SS}=2)$, pterygoid mandibular space $(\mathrm{SS}=2)$ and lateral pharyngeal space $(\mathrm{SS}=3)$ - thus yielding a total of 11 points out of a maximum of 36 points. In our opinion, this classification alone is unable to offer a clear idea of the true severity of the infection, since the sum obtained did not even reach half of the maximum score.

The most frequent cause of death in patients with OI is respiratory tract obstruction $(5,7)$. The physician therefore must evaluate this aspect at initial patient assessment. It is of great importance to identify certain signs and symptoms when anatomical spaces are compromised (Table 1).

Trismus is an obvious sign suggestive of serious OI. Buccal opening that has diminished $20 \mathrm{~mm}$ or more in a short period of time, with severe pain, is considered to indicate infection of the perimandibular anatomical spaces until proven otherwise $(2,8,10)$. Nonetheless, regardless of trismus, the attending physician must assess the presence of dysphagia and visualize the oropharynx in search of a possible infectious process.

In cases of partial obstruction of the respiratory tract, abnormal sounds will be heard, such as stridency and 
Table 1. Severity scores for severe odontogenic infections according to anatomical space involvement. (Reproduced from Flynn et al. Severe Odontogenic Infections. J Oral Maxillofac Surg 2006).

\begin{tabular}{|l|c|}
\hline Severity score & Anatomical space involved \\
\hline $\begin{array}{l}\text { Severity score }=1 \\
\text { (Mild risk for airway and/or vital structures) }\end{array}$ & Vestibular \\
& Subperiosteal \\
& Space of the body of the mandible \\
Infraorbital \\
Buccal
\end{tabular}

NOTE: The severity score for a given subject is the sum of the severity scores for all of the spaces affected by cellulitis or with abscesses, based on the clinical and radiographic findings.

wheezing, due to the turbulent passing of air through the respiratory tract. In these cases the patient typically inclines the head frontward or moves the neck towards the opposite shoulder in order to straighten the respiratory tract and thus improve ventilation (10). Oxygen saturation below $94 \%$ in a previously healthy patient is a sign of insufficient oxygenation of the tissues. When accompanied by clinical signs of partial or total obstruction, it constitutes a surgical emergency, and urgent endotracheal intubation must be performed in order to secure the respiratory tract via a tracheotomy or cricothyrotomy as in our case.

In several studies $(2,4,8)$, the initial leukocyte count has been cited as an important predictor during hospital admission. Leukocytosis above 12,000 cells $/ \mathrm{mm}^{3}$ generates a systemic inflammatory response syndrome (SIRS), which is an important factor in determining hospital admission due to OI (13). In $66 \%$ of their cases, Flynn et $a l$. were able to correlate hospital stay to the severity scale values and leukocyte counts at hospital admission. In our patient the leukocyte count upon admission was 20,000 cells $/ \mathrm{mm}^{3}$, with fever $\left(38.5^{\circ} \mathrm{C}\right)$, causing an increase in metabolic and cardiovascular demand beyond the reserve capacity, where loss of fluids is greatly increased and entails severe dehydration.

Certain medical conditions can interfere with functions of the immune system that are essential for host defense against OI. Diabetes mellitus (DM) is the most prevalent chronic disease affecting the immune system $(7,9)$ - hyperglycemia being the main etiological factor of DM leading to dysfunction of the immune system. All the main immune cell types are affected. In this regard, neutrophil adhesion, chemotaxis and phagocytosis are altered (10-12), and this results in a less efficient defense against microbial attack (13).

Diabetes is also characterized by exacerbated macrophage reactions, which increase the production of proinflammatory cytokines and intensify connective tissue metalloproteinase response - resulting in difficulties for containing infection (9). On the other hand, chronic hyperglycemia can exert an influence upon fibroblast proliferation and collagen synthesis, hindering tissue replacement and wound healing $(9,10)$.

Our patient presented a glycosylated hemoglobin (HbAlc) value of $17 \%$, consistent with the lack of disease follow-up over the last 12 months. This poor metabolic control increased patient susceptibility to immune system alterations. Hospital stay in diabetic patients with $\mathrm{OI}$ is much longer than in non-diabetic patients, and head and neck space involvement is also more frequent than in non-diabetic individuals. A strong link therefore exists between diabetes and complications in the management of severe OI (7-9).

The place where the patient should be operated upon (i.e., in a specialized center or on an outpatient basis) is decided from the data obtained at initial examination $(7,8)$. In our case, the patient suffered decompensated systemic disorders such as DM, and presented clinical characteristics confirming the seriousness of OI. Accordingly, emphasis was placed on compensating the basal disease conditions before performing surgery (13). Regardless of the severity of OI, surgical management is 
based on two principles: elimination of the causal focal point of infection, and surgical voiding of the compromised anatomical spaces together with adequate drainage (10-12). Surgical management of the compromised anatomical spaces must be made aggressively and promptly, as initially described by William and Guralnick (14). This approach is based on the concept that prompt emptying and surgical drainage nullifies the propagation of infection towards deeper and more severe spaces, even if the infection is in a phlegmon state (13). Samples for microbiological culture and antibiogram can be obtained at this stage. However, since the results take some time in becoming available, this practice should be reserved for cases where OI affects multiple spaces, or in patients with immune system alterations $(2,7)$. In our patient surgery was performed on day four of hospital admission, once the basal disease conditions had been evaluated and the airway had been secured, and consisted of elimination of the causal focal point of infection and extensive cervicotomy.

\section{References}

1. Poveda-Roda R, Bagan JV, Sanchis-Bielsa JM, Carbonell-Pastor E. Antibiotic use in dental practice: a review. Med oral Patol oral cir Bucal. 2007;12:186-92.

2. Flynn TR, Shanti RM, Levi MH, Adamo AK, Kraut RA, Trieger N. Severe odontogenic infections, part 1: prospective report. J Oral Maxillofac Surg. 2006;64:1093-103.

3. Jaunay $T$, Sambrook P, Goss A. Antibiotic prescribing practices by South Australian general dental practitioners. Aust Dent J. 2000;45:179-86.

4. Flynn TR, Wiltz M, Adamo AK, Levy M, McKitnick J, Freeman K. Predicting length of hospital stay and pencillin failure in severe odontogenic infections. Int J oral Maxillofac Surg. 1999;28:48.

5. Zamiri BA, Hashemi SB, Hashemi SH, Rafiee ZC, Ehsani S. Prevalence of Odontogenic Deep Head and Neck Spaces Infection and its Correlation with Length of Hospital Stay. Journal of Dentistry, Shiraz University of Medical Sciences. 2012;13:29-35.

6. Umeda M, Minamikawa T, Komatsubara H, Shibuya Y, Yokoo S, Komori T. Necrotizing fasciitis caused by dental infection: a retrospective analysis of 9 cases and a review of the literature. Oral Surg Oral Med Oral Pathol Oral Radiol Endod. 2003;95:283-90.

7. Kim MK, Nalliah RP, Lee MK, Allareddy V. Factors associated with length of stay and hospital charges for patients hospitalized with mouth cellulitis. Oral Surg. Oral Med. Oral Pathol. Oral Radiol. 2012;113:21-8.

8. Flynn TR, Shanti RM, Hayes C. Severe Odontogenic Infections, Part 2: Prospective Outcomes Study. J Oral Maxillofac Surg. 2006;64:1104-13

9. Kamat RD, Dhupar V, Akkara F, Shetye O. A comparative analysis of odontogenic maxillofacial infections in diabetic and nondiabetic patients: an institutional study. Journal of the Korean Association of Oral and Maxillofacial Surgeons. 2015;41:176-80.

10. Jiménez Y, Bagán JV, Murillo J, Poveda R. Odontogenic infections. Complications. Systemic manifestations. Med Oral Patol Oral Cir Bucal. 2004;9:143-7,139-43.

11. Lugo AFG, Ravago MGC, Martinez RAG, Peltrini RJZ. Ludwig's angina: A report of two cases. Revista Española de Cirugía Oral y Maxilofacial. 2014;36:177-81.

12. Velasco I, Soto R. Principles for the treatment of odontogenic infections with different levels of complexity. Revista chilena de cirugía. 2012;64:586-98

13. Kinzer S, Pfeiffer J, Becker S, Ridder GJ. Severe deep neck space infections and mediastinitis of odontogenic origin: clinical relevan- ce and implications for diagnosis and treatment. Acta otolaryngol. 2009; 129:62-70.

14. Williams AC, Guralnick WC. The diagnosis and treatment of Ludwig's angina: A report of twenty cases. New England Journal of Medicine. 1943;228:443-450.

15. Kurien M, Mathew J, Job A, Zacharia N. Ludwig's angina. Clin Otolaryngol Allied Sci. 1997;22:263-5.

\section{Conflict of Interest}

The authors have declared that no conflict of interest exist. 